\title{
Estimated Impact of Public and Private Sector COVID- 19 Diagnostics and Treatments on US Healthcare Resource Utilization
}

\author{
Daniel M. Sheinson - William B. Wong · Caroline E. Solon • \\ Mindy M. Cheng $\cdot$ Anuj Shah $\cdot$ David Elsea $\cdot$ Yang Meng
}

Received: October 28, 2020 / Accepted: December 4, 2020 / Published online: December 26, 2020

(C) The Author(s) 2020

\begin{abstract}
Introduction: Coronavirus disease 2019 (COVID-19) has imposed a considerable burden on the United States (US) health system, with particular concern over healthcare capacity constraints.

Methods: We modeled the impact of public and private sector contributions to developing diagnostic testing and treatments on COVID19-related healthcare resource use.

Results: We estimated that public sector contributions led to at least $30 \%$ reductions in COVID-19-related healthcare resource utilization. Private sector contributions to expanded diagnostic testing and treatments led to further reductions in mortality $(-44 \%)$, intensive care unit (ICU) and non-ICU hospital beds (- 30\%
\end{abstract}

Supplementary Information The online version contains supplementary material available at https:// doi.org/10.1007/s12325-020-01597-3.

D. M. Sheinson $(\bowtie) \cdot$ W. B. Wong .

C. E. Solon · A. Shah

Genentech, Medical Affairs, South San Francisco,

CA, USA

e-mail: sheinson.daniel@gene.com

M. M. Cheng

Roche Molecular Systems, Inc, Global Access and

Health Economics, Pleasanton, CA, USA

D. Elsea · Y. Meng

Bresmed, Health Economic Analysis, Las Vegas, NV, USA and $-28 \%$, respectively), and ventilator use (-29\%). The combination of lower diagnostic test sensitivity and proportions of patients selfisolating may exacerbate case numbers, and policies that encourage self-isolating should be considered.

Conclusion: While mechanisms exist to facilitate research, development, and patient access to diagnostic testing, future policies should focus on ensuring equitable patient access to both diagnostic testing and treatments that, in turn, will alleviate COVID-19-related resource constraints.

Keywords: COVID-19; Diagnostic test; Health policy; Health resources 


\section{Key Summary Points}

Why carry out this study?

A compartmental model for COVID-19related disease transmission, healthcare resource utilization, and mortality was calibrated to observed COVID-19 tracking data in order to study the impact of treatment and testing during the pandemic in the USA

To what extent have novel treatments and expanded diagnostic testing alleviated the severity of the COVID-19 pandemic in the USA in terms of resource use, disease transmission, and mortality?

\section{What was learned from the study?}

Both public and private sector contributions to diagnostic testing and treatment were estimated to have led to a reduction in COVID-19 cases, mortality, and hospital bed/ventilator utilization; thus, policies which facilitate equitable access to testing and treatments are warranted

Our findings suggest that false negatives may contribute significantly to increases in cases, particularly in conjunction with poor self-isolation; thus testing accuracy should be considered in combination with time to test results

A combination of treatments which alter different outcomes, balancing reductions in mortality with reductions in hospital length of stay, may be optimal to provide health system capacity relief; thus, policies which facilitate continued innovation in treatments are critical

\section{DIGITAL FEATURES}

This article is published with digital features, including a summary slide, to facilitate understanding of the article. To view digital features for this article go to https://doi.org/10. 6084/m9.figshare.13325630.

\section{INTRODUCTION}

As of September 23, 2020, over 30 million coronavirus disease 2019 (COVID-19) cases are estimated to have occurred worldwide, resulting in more than 900,000 deaths [1]. Of these, the United States (USA) is estimated to account for over 6 million cases and more than 200,000 deaths. Despite constantly evolving data as the pandemic progresses, early estimates suggest that approximately $20 \%$ of COVID-19-infected patients require hospitalization [2]. Of adults hospitalized in the USA, estimates suggest that $32 \%$ required intensive care unit (ICU) admission and $19 \%$ required invasive mechanical ventilation [3], representing a considerable burden to the health system.

Health system capacity has been a widely reported concern in the USA since the onset of the pandemic, with many studies highlighting an insufficient number of hospital beds, ICU beds, and mechanical ventilators to address the additional needs anticipated with COVID-19 [4-6]. The dramatic increase in need for healthcare resources by patients with COVID-19 and severe manifestations that require more intensive treatment risks overwhelming hospital systems, in terms of both staff and space to treat patients [5]. For example, the New York Public Health System had a pre-pandemic capacity of 300 ICU beds, yet at the peak of the COVID-19 surge were caring for 1000 ICU patients [7]. This burden is further compounded by the longer lengths of stay (LOS) required by patients with COVID-19 requiring respiratory support, with estimates of ICU ventilator use ranging from 18 to 23 days vs. approximately 3-8 days during non-pandemic times [8-10]. In turn, capacity constraints-particularly in the ICU setting-may result in increased mortality rates [11-14].

Early models focused on estimating the impact of COVID-19 on hospital system capacity metrics, including admissions, ICU admissions, and mechanical ventilator use $[6,15]$. 
However, since then, as a result of the complex challenges of COVID-19, both the public and private sectors have contributed significantly to increasing diagnostic testing capacity, beyond public health laboratories and individual laboratory developed tests (LDTs), and studying treatments to address COVID-19. For example, in April 2020, the Accelerating COVID-19 Therapeutic Interventions and Vaccines (ACTIV) partnership was formed between members of the public and private sectors to develop a coordinated research strategy to help accelerate the development and distribution of COVID-19 therapeutics and vaccines [16]. Given the rapid development of innovations to address COVID-19, this presents an opportunity to develop models focusing specifically on the impacts of diagnostic testing and therapeutic intervention that have not been accounted for in previous models.

Despite both the public and private sectors accelerating diagnostic testing and treatments through Food \& Drug Administration (FDA) emergency use authorizations (EUAs), there remains a large degree of uncertainty regarding the impact of COVID-19 testing and treatment at reducing time to recovery, ICU or ventilator use, and/or mortality. Therefore, the objective of this research was to develop a model to estimate the impact to date of public and private sector contributions to developing effective COVID-19 treatment and nucleic acid-based (molecular) diagnostic testing on resource use in the hospital (including hospital beds, ICU beds, and mechanical ventilators) and overall population impacts (including mortality and cumulative incidence of disease) at the US national level during the pandemic.

\section{METHODS}

\section{Model Structure}

The model described in this article leverages data from previously conducted studies and does not contain any studies with human participants or animals performed by any of the authors.
An age-stratified compartmental model of disease transmission and healthcare resource utilization across the entire USA was adapted from Moghadas et al. [6] and further developed by incorporating the effects of diagnostic testing among infected individuals and novel treatment in the hospital setting. While the transmission rates in the USA have been shown to vary by geographic region, a model at this level would require region-specific details around testing and treatment capacity, which are not publicly available. Hence, we aimed to estimate disease spread and resource use in aggregate across the country (see "Discussion" section for limitations). In our model, individuals start as uninfected but susceptible to disease and potentially become exposed to infection according to a time-varying transmission rate. Once exposed, individuals develop asymptomatic, mild, or severe disease. A subset of symptomatic individuals self-isolate, thereby reducing their contact rate with others in the population and the overall transmission of infection. Asymptomatic and mildly symptomatic individuals were assumed to recover from infection while a proportion of severely symptomatic individuals incur different levels of hospital resource utilization in the form of general ward (non-ICU), ICU admission, or ICU admission with mechanical ventilation (Fig. 1). Hospitalized patients either recover or die according to separate rates of recovery and mortality for each level of care. Recovered individuals were assumed to be immune and not infect others. Four age categories (0-19, 20-49, 50-64, 65+) were modeled throughout, allowing for age-specific transition probabilities of developing severe illness, hospitalization, and ICU admission. The model was implemented in Microsoft Excel using a Markov structure with 1-day cycles and was initialized by assuming one exposed individual in each age group at day 0 corresponding to January 21, 2020.

Diagnostic testing was incorporated into the model by assuming that some infected individuals undergo testing, upon which a positive test result leads to self-isolation of that individual. Treatment was incorporated into the model through specific inputs affecting the 


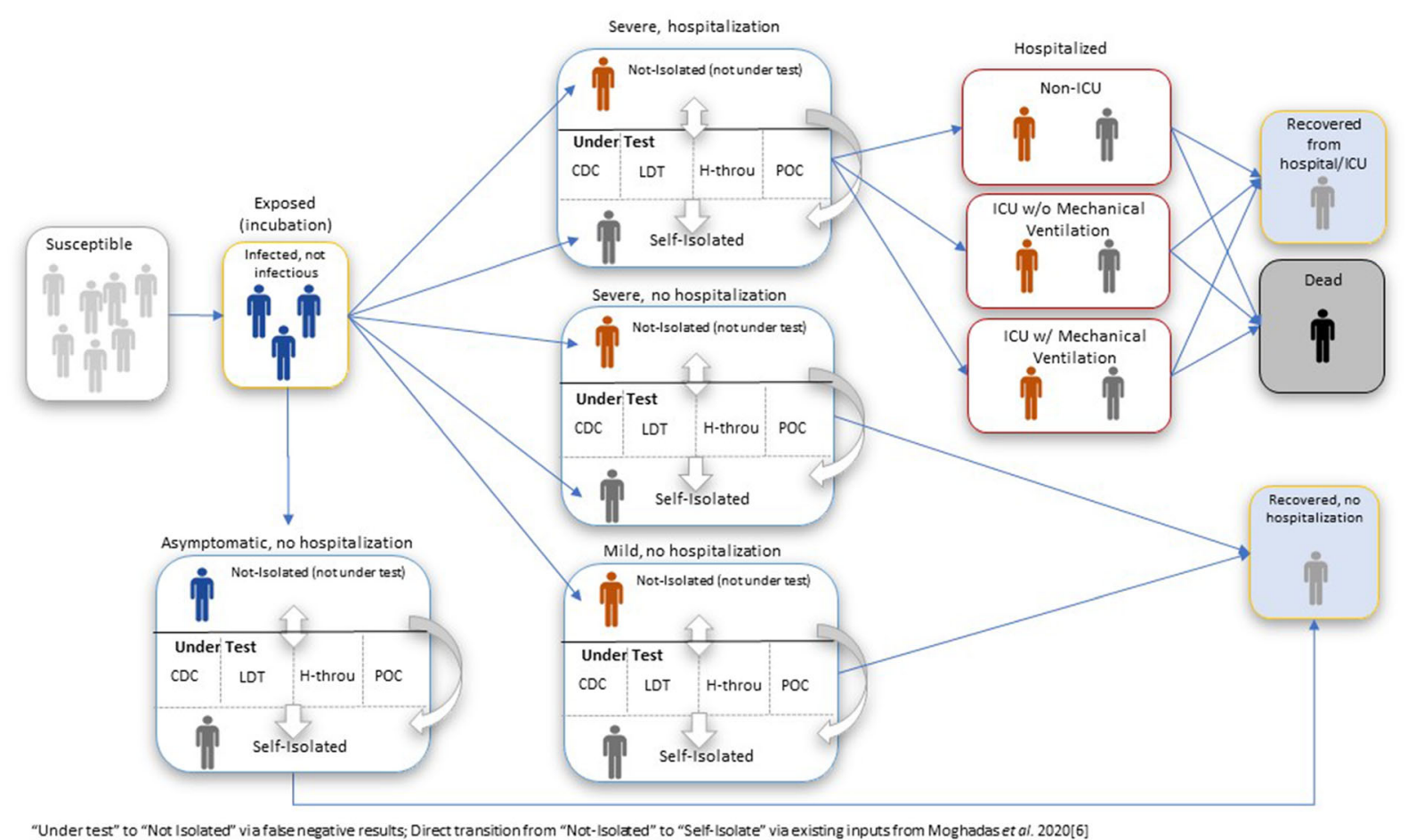

Fig. 1 Compartmental model structure

rates of hospital recovery, mortality, and mechanical ventilation usage. Additional model input parameters control the size and age distribution of the population, rates of transmission and recovery, rates of testing and time to results, symptom onset and severity, rates of self-isolation, levels of hospitalization, mortality, and testing capacity.

\section{Model Calibration and Data Sources}

Published literature, government, and nongovernment sources on COVID-19 were gathered to inform input parameter values and provide prior information for calibrating the model to observed data from The COVID Tracking Project [17] (see appendix in electronic supplementary materials for detailed methodology). Model parameters related to disease transmission, symptoms, self-isolation, and testing were obtained mainly from early publications on the spread of the disease in China $[18,19]$ and input into the model directly (see appendix Tables S1 and S2 in the electronic supplementary materials for details). Model parameters related to hospitalization, including hospitalization rate, proportion admitted by care setting, and rates of death and recovery were obtained mainly from US-specific sources and informed prior distributions for calibrating those parameters to the observed data (see appendix Table S4). In addition, time-varying rates of transmission and testing were incorporated into the model and-along with the aforementioned hospitalization parameterscalibrated to the observed number of daily positive tests, patients in the hospital, patients in the ICU, patients on mechanical ventilation, and cumulative deaths using a sequential estimation technique called the kernel density particle filter [20]. Model calibration was implemented using R version 3.5.3 [21], and the programming code is available on GitHub (https://github.com/Roche/covid-hcru-model). 


\section{Model Scenarios}

Once the model was calibrated, we simulated five scenarios to compare expected transmission, hospital resource use, and mortality: (1) a reference scenario reflecting no public or private sector contributions to diagnostic testing or treatment; (2) a public sector only scenario reflecting diagnostic testing and treatment contributions from the public sector; (3) a scenario with public and private sector contributions in developing effective treatment only; (4) a scenario with public and private sector contributions in developing effective diagnostic testing only; (5) a scenario with public and private sector contributions in developing both effective treatment and testing. Each scenario was generated using modified input parameters reflecting the assumed level of testing and treatment in the population. Model results for each scenario are reported for a time period when private sector diagnostic tests and effective novel treatments were both available via FDA EUAs. This was defined to be from June 1, 2020 to the latest date where COVID-19 tracking data were available at the time of model calibration (August 21, 2020).

\section{Molecular Diagnostic Testing}

Test systems considered in the model included commercial high-throughput (HT) molecular assays (as defined in the Centers for Medicare and Medicaid Services [CMS] Ruling 2020-01-R as of April 14, 2020 [22]) and molecular pointof-care (POC) assays, which received FDA EUA by June 1, 2020; other commercial tests (Other); a test developed by the Centers for Disease Control and Prevention (CDC); and independent LDTs. In the public sector only scenario, it was assumed that only LDTs and the CDC test for SARS-CoV-2 diagnosis would be available (i.e., no HT or POC tests available). In scenarios that included the effects of test systems developed through the private sector, the capacity of HT, POC, and other commercial tests were set according to market projections based on publicly available data reported by major test manufacturers. Market share assumptions for the different test systems were based on survey data [23] on their relative use in laboratories.

\section{Treatment Efficacy}

Given its established availability prior to the pandemic and the RECOVERY study [24], which demonstrated its clinical benefit for COVID-19, and being funded primarily via ex US sources, dexamethasone was assumed to be an available treatment option in the public sector only scenario. In the scenarios including treatments developed from the private sector, treatment effects on hospital recovery and mortality rates were based on data for remdesivir since it was the only new treatment that was issued an EUA by the FDA for COVID-19 [25] at the time of model calibration. Given the recent data suggesting that there may be some uncertainty surrounding the efficacy of remdesivir [26, 27], we conducted a number of sensitivity analyses (see "Scenario and Sensitivity Analyses"). To be conservative with respect to some eligible patients not receiving treatment or discontinuing their treatment early, it was assumed that $50 \%$ of eligible patients were treated.

\section{Scenario and Sensitivity Analyses}

Additional scenario analyses were conducted to explore the inclusion of additional treatment effects on mortality and reduced use of mechanical ventilation. Given the potential uncertainty in the treatment effects of remdesivir $[26,27]$, a scenario analysis was also conducted for which the private sector effects were due to testing only (i.e., remdesivir treatment effects were excluded). One-way and two-way sensitivity analyses were conducted to quantify the impacts of uncertainty associated with individual model parameters, including the market share of HT testing, the time at which public-private testing and treatment were available, and the impact of test sensitivity versus the proportion of patients that self-isolate while awaiting their test results. 


\section{Model Availability}

While this model is based on historical data, for those wishing to estimate custom scenarios for healthcare capacity planning, particularly as new advancements in the diagnosis and care of patients with COVID-19 become available, the model is available in the electronic supplementary material.

\section{RESULTS}

Calibration of model parameters resulted in estimated resource utilization and cumulative mortality that tracked with observed data from The COVID Tracking Project (Fig. 2). In the absence of public and private sector contributions (reference scenario), we estimated a cumulative incidence of 17,952,264 COVID-19 cases, a cumulative mortality of 315,230 deaths, peak (i.e., maximum) non-ICU hospital beds needed of 153,698, peak ICU beds needed of 37,245 , and peak ventilator use of 15,338 ventilators from June 1 to August 21, 2020 in the USA (Table 1; Fig. 3a-d). In the public sector only scenario, which assumed availability of only the CDC test and LDTs without novel effective treatments attributed to the private sector, we estimated at least $30 \%$ reductions in all outcomes measured relative to the reference scenario, with the largest effect on cumulative mortality $(-40.2 \%)$. The addition of private sector contributions to diagnostic testing capacity and effective treatment options were associated with additional reductions (relative to the public sector only scenario) across all outcomes, with the greatest effect on cumulative mortality $(-44.0 \%)$ and reductions on other outcomes ranging from $-15.2 \%$ to $-29.9 \%$ (Table 1).

Examining the individual effects of the private sector contributions allows us to infer the relative contributions of expanded diagnostic testing versus novel treatment that contribute to reductions in individual outcomes. The public + private sector scenario assumed that novel treatment from the private sector
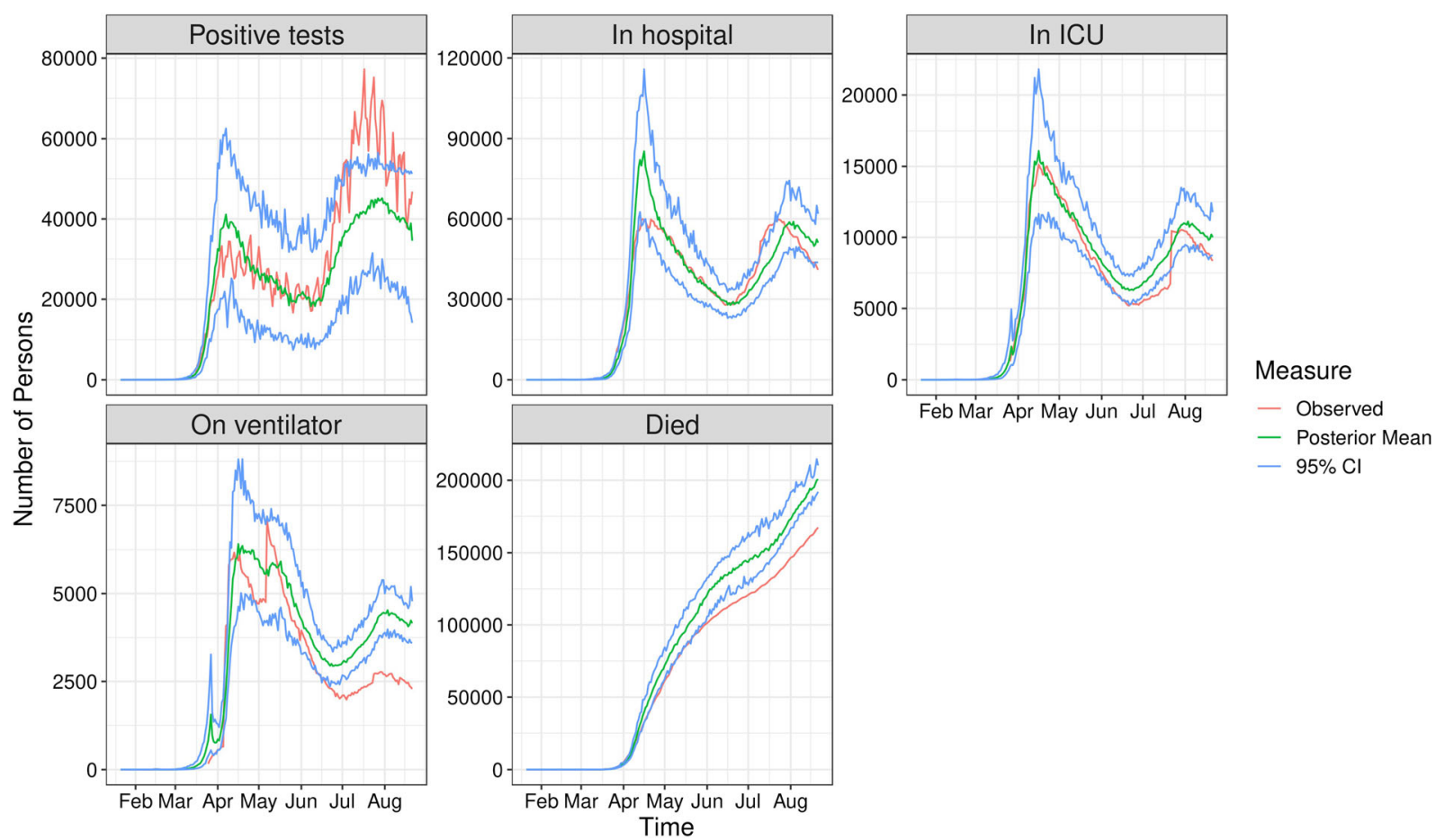

Fig. 2 Estimated resource utilization (posterior means) over time and $95 \%$ credible intervals from calibrated model alongside observed data from The COVID Tracking Project 


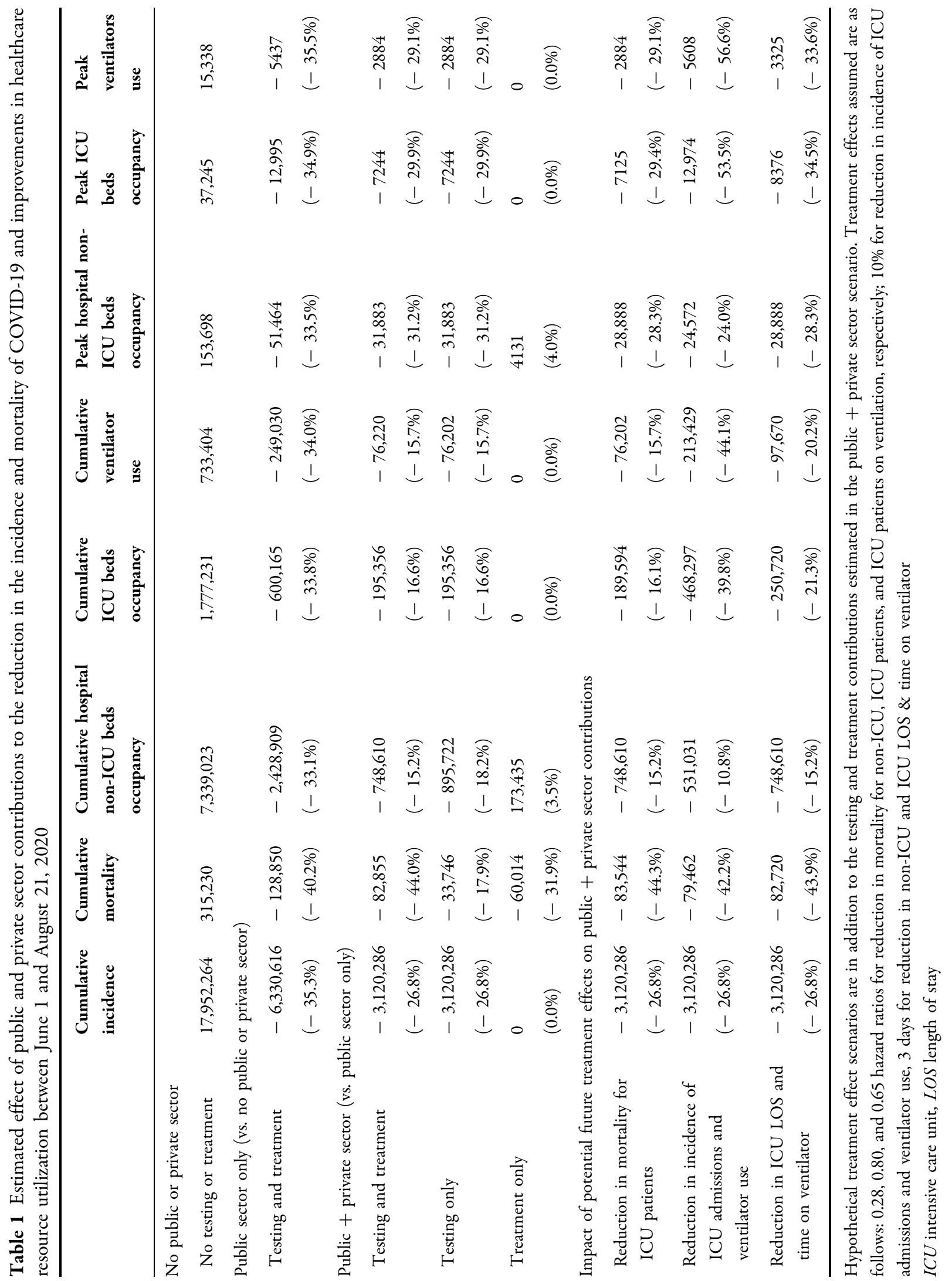



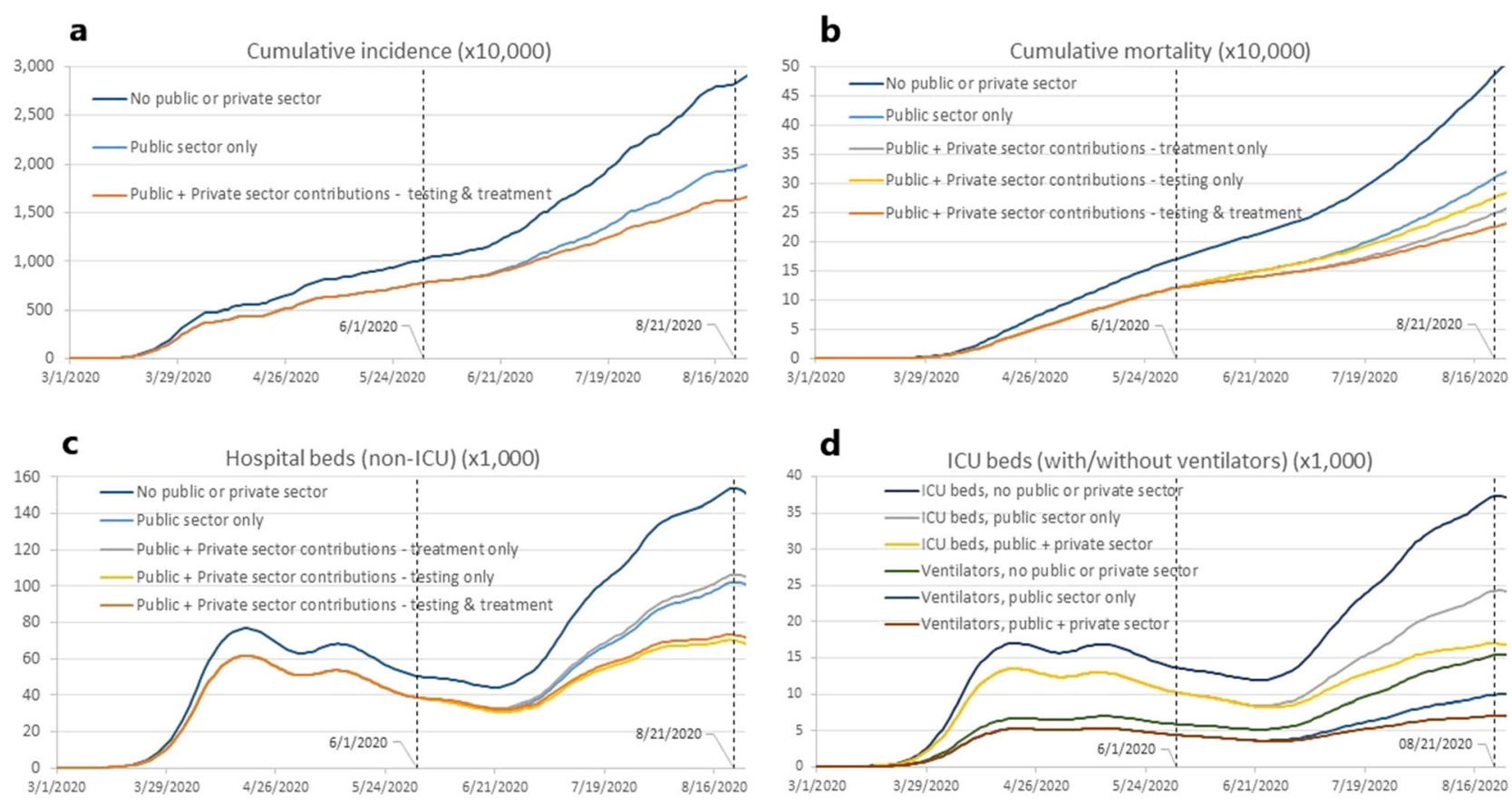

Fig. 3 Public and private sector contributions for COVID-19 incidence (a) and mortality (b) and hospital resource use over time (c, d). 6/1/2020 is the assumed start date of the private sector contributions to diagnostic

testing and novel effective treatments. $8 / 21 / 20$ is the end date of the results reporting period. ICU intensive care unit

provided only a mortality benefit and reduced LOS among non-ICU hospitalized patients. Therefore, the scenario with novel effective treatment alone only affected cumulative mortality $(60,014$ fewer deaths; $-31.9 \%)$, cumulative non-ICU hospital occupancy $(173,435$ more beds; $+3.5 \%)$, and peak non-ICU hospital occupancy (4131 more beds; $+4.0 \%)$. In this case, the mortality benefit leads to greater nonICU hospital resource use due to longer LOS among non-ICU patients that recover compared to those that die.

The individual effect of private sector expansion of commercial diagnostic testing capacity alone (i.e., excluding any private sector treatment effects) affected all outcomes. This was due to the increased number of true positive test results leading to greater self-isolation and reduced transmission, which has downstream effects on hospital resource use and mortality. In this scenario, expanded testing contributed to $3,120,286$ fewer cases $(-26.8 \%)$, 33,746 fewer deaths $(-17.9 \%), 31,883$ fewer

non-ICU beds at peak occupancy (-31.2\%), 7244 fewer ICU beds at peak occupancy (-29.9\%), and 2884 fewer ICU patients on mechanical ventilation at peak usage $(-29.2 \%)$. Taken together, we can infer that for the combined effects, expanded diagnostic testing was the larger contributor to reducing non-ICU hospital bed occupancy while effective novel treatment had a greater contribution to reducing mortality.

Scenario analyses examining the various effects of treatments, as a proxy for potential new treatments which may have different effects than those currently marketed, demonstrated a potential reduction in outcomes not previously impacted by current treatment via public and private sector contributions (Table 1). When treatment-related reductions in ICU admissions and ventilator use were applied, the estimated reduction in peak ICU beds increased 1.8 -fold (-53.5\% vs. $-29.9 \%$ ) and cumulative ICU beds increased 2.4-fold $(-39.8 \%$ vs. $-16.6 \%)$. Similarly, assuming 


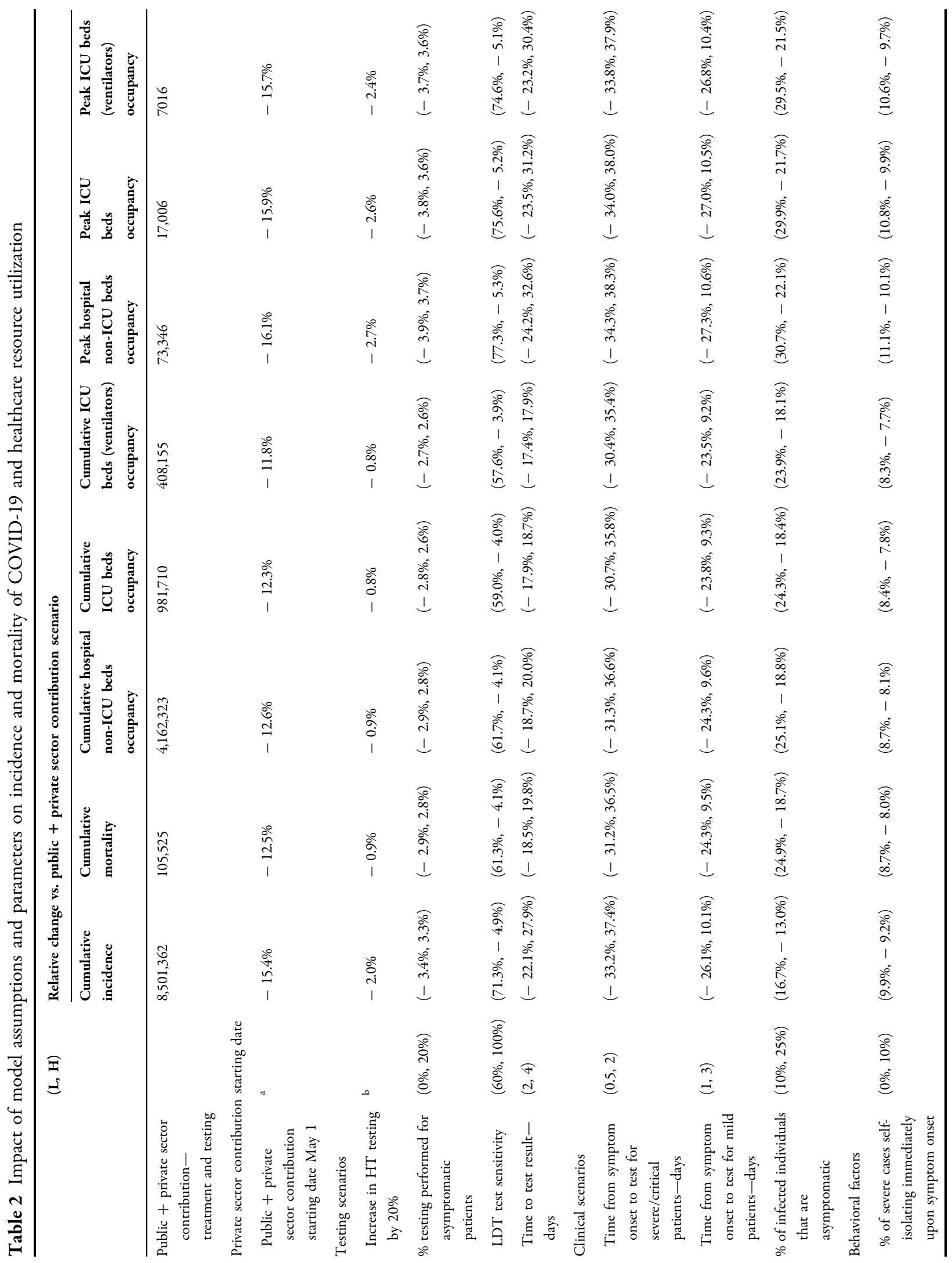


reductions in ICU LOS and time on ventilator further reduced peak and cumulative ICU beds but to a lesser extent ( $-34.5 \%$ vs. $-29.9 \%$, $-21.3 \%$ vs. $-16.6 \%)$.

In sensitivity analyses, changing the date at which expanded diagnostic testing and novel treatment were assumed to be available from June 1 to May 1 (as a proxy for when remdesivir was first available via EUA) resulted in further reductions in resource utilization, mortality, and incidence of COVID-19 cases (range $-11.8 \%$ to $-16.1 \%$; Table 2 ). Among the diagnostic testing parameters varied, the model was highly sensitive to assumptions around time to test result and LDT sensitivity. Increasing time to test result by just 1 day (from 3 to 4 days) increased the cumulative incidence of cases by $+27.9 \%$ and resources utilized by +17.9 to $+32.6 \%$. Similarly, varying LDT sensitivity from $100 \%$ to as low as $60 \%$ resulted in large changes in the incidence of COVID-19 cases $(-4.9 \%$ to $+71.3 \%$ cases $)$ and resource use (ranging from $-5.3 \%$ to $+77.3 \%$ ). Shifting $20 \%$ of the share of available testing systems toward more HT tests resulted in changes in all outcomes ranging from $-0.8 \%$ to $-2.7 \%$. Patient compliance with self-isolation while awaiting test results was also found to have a significant effect on the cumulative incidence of cases and accentuated the effects of lower testing accuracy (Fig. 4). For example, the change in cumulative incidence of COVID-19 cases for diagnostic test sensitivities ranging from $100 \%$ to $85 \%$ was between $-10.2 \%$ and $+50.0 \%$ for the assumed level of $70 \%$ self-isolation while awaiting test results. However, when self-isolation while awaiting test results was decreased to $50 \%$, the change in cumulative incidence of COVID-19 cases ranged from $+219.8 \%$ to $+340.3 \%$. Model sensitivity to other clinical and behavioral factors is $s$ hown in Table 2.

\section{DISCUSSION}

To our knowledge, this is the first study to comprehensively estimate public and private sector contributions, via development of molecular diagnostic testing and treatments, in 


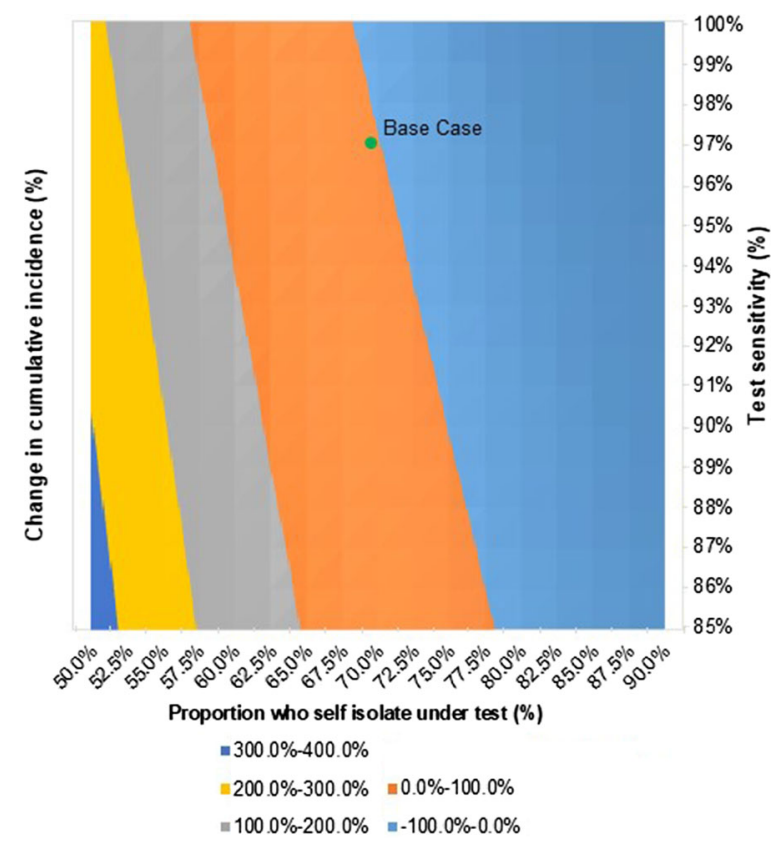

Fig. 4 The relationship between test sensitivity and selfisolation while awaiting test results on cumulative incidence of COVID-19 cases. Results displayed are the percentage change in cumulative incidence relative to the scenario assuming public + private sector contributions to diagnostic testing and novel treatment

addressing COVID-19-related health system resource constraints in the USA. We found that the effect of public sector contributions and EUAs expanding and expediting the availability of COVID-19 commercial diagnostic tests and therapeutics likely had a significant effect on altering the trajectories of cases, mortality, and resource utilization. This finding is consistent despite uncertainty in private sector treatment effects, indicating that diagnostics alone may have a role in reducing health resource utilization, although the combination of both testing and treatments together produced a greater effect than either considered alone. While this model focuses on the cases and resource use from a US national perspective, regional differences in COVID-19 have been well documented. Since the availability of testing and treatment resources may vary regionally and may not align with the number of cases present in a particular geography, our model provides an optimistic scenario and highlights the importance of ensuring efficient and equitable distribution of treatments and diagnostics.

While this study focused on resource utilization, it is also important to consider the economic implications of resource reductions. Estimated COVID-19 healthcare costs per day to hospitals range from $\$ 2303$ (non-ICU) to $\$ 3449$ (ICU with ventilation) [28]. With hospitals facing substantial financial burden due to COVID19 , any diagnostic test or treatment which can reduce hospitalizations and LOS may provide significant cost savings. While estimating costs was beyond the scope of this study, it should be noted that the costs of a course of treatment for the only guideline-recommended COVID-19 treatments during this study period (remdesivir and dexamethasone) [29-31] are less than, or similar to, a single day in a hospital.

On the basis of our findings, public and private sector contributions have played an important role in addressing the pandemic; however, critical needs remain. Despite the increase in diagnostic testing capacity over time, laboratory backlogs have been observed [32]. As estimated in this study, time to test result is an important factor in reducing laboratory backlog and influences the infection curve and hospitalizations. Because of the urgency to increase testing capacity, other factors, including test sensitivity, have been less scrutinized. Our findings highlight that test sensitivity is an important consideration. In particular, the real-world clinical validity of available test options is largely unknown, and our results demonstrate that tests with lower sensitivity (i.e., increased false negative results) may lead to higher risk of viral transmission and increase healthcare resource utilization. Therefore, it is important to consider test system capacity, time to test result, and test sensitivity when evaluating the potential effectiveness of different testing strategies. Additionally, behavioral factors, such as patient compliance with self-isolation, are crucial for minimizing viral transmission. We found that the combination of poor patient self-isolation behaviors and lower test sensitivity can exacerbate the impact of false negatives on disease transmission and subsequent resource utilization. Therefore, when considering trade-offs between 
test sensitivity and time to test result, patient compliance with self-isolation behaviors is an important parameter to understand. Consequently, policies that support and enable people to self-isolate without penalties or risks (e.g., loss of employment, school, etc.) are important.

Effective treatments are equally important given the complementary effects of diagnostic testing on COVID-19 healthcare resource use and patient outcomes. Recent therapies issued EUA have involved both private sector contributions (e.g., remdesivir) and public funding (e.g., dexamethasone, convalescent plasma). We projected that the treatments (remdesivir, dexamethasone) have impacted mortality and non-ICU hospital resource use. Research and development (R\&D) is occurring at a record pace, with over 300 therapies under investigation for COVID-19 [33]. These present potential opportunities to further reduce capacity constraints. This may be particularly important when considering individual treatment effects, since those that reduce mortality may increase hospital LOS because of the prolonged survival effect. This was the case in our study, where the effect of diagnostic testing alone led to a greater reduction in non-ICU beds than with testing and treatment. Therefore, a potentially optimal scenario may be a combination of novel treatments that balance the reduction in mortality and LOS outcomes. Production and distribution of any future vaccine at scale may be challenging; thus, development of novel treatments may have similar or even greater importance than vaccine development. However, unlike the rapid progress in diagnostic testing innovation, developing therapeutics has been more challenging, with many failed trials highlighting the difficulty of finding R\&D success. Policies that continue to facilitate $R \& D$ are critical for the ability to develop innovative approaches to addressing the COVID-19 pandemic.

In addition to policies that facilitate $R \& D$ and allow recent innovations to be quickly available, those that enable patient access to these innovations are equally important. For example, the Coronavirus Aid, Relief and Economic Security (CARES) Act allows coverage of testing without cost-sharing, including those receiving testing out of network [34]. The significant impact diagnostic testing may have on multiple aspects of healthcare resource utilization, as observed in this study, highlights the importance of policies that facilitate patient access to testing resources. However, despite the enactment of these policies, there may still be subgroups of patients who are not covered by the CARES Act, such as the uninsured. Given rapid developments in the availability of testing technologies (e.g., antibody, antigen, multiplex molecular tests, etc.), the impact of testing on resource utilization, and current understanding that lower socioeconomic status patientsmany who may be uninsured-are disproportionately affected by COVID-19 [35-37], policies that clarify coverage requirements and facilitate patient access to all COVID-19 testing technologies should be considered.

Unlike with testing, there is currently a lack of federal policies facilitating access to COVID19 treatment via limiting cost-sharing. We found that treatment contributed a larger portion of the reduction in mortality relative to diagnostic testing, highlighting the importance of access to effective treatments. While many private payers have waived cost sharing for their members [38], some patients may still be vulnerable to high cost-sharing responsibilities, such as those with high deductible insurance plans (that have not waived cost sharing) or the uninsured. These patients may delay seeking care because of cost, resulting in additional resource utilization due to missed opportunities to leverage future/existing treatments at an earlier disease stage. Furthermore, delays or avoiding care may result in greater mortality $[39,40]$ and downstream productivity losses, accentuating the existing disparities in care. Future policies that ensure equitable access to hospital care and treatment should be considered.

Like all models, ours has limitations. First, the model assumes equal distribution of treatments and testing across the USA. In reality, healthcare resources in the USA are not equally distributed relative to need, so the impact of diagnostics and treatments may vary by individual health system. Thus, estimates of expected resource use relative to known availability of hospital/ICU beds and ventilators throughout 
the country should only be interpreted in aggregate across the country and are not necessarily reflective of the resource burden faced by individual health systems. Second, expanded testing scenarios assume that laboratory infrastructures are in place and the necessary consumables are widely available when this may not be the case. Lastly, the model assumes that treatments and testing became available instantaneously on a single date when in actuality the availability of new diagnostics and treatments was spread out over time. This may, for example, overestimate the impact of public sector contributions, as it is assumed that both public sector testing capacity and treatments (dexamethasone) were available at scale since the beginning of the pandemic.

\section{CONCLUSION}

Public and private sector efforts have provided substantial contributions to reducing COVID19-related transmission, healthcare resource utilization, and mortality. Both diagnostic testing capacity and accuracy are important aspects to consider when identifying how to optimally deploy testing resources. Current COVID-19 treatment options are limited, yet provide significant contributions to reducing healthcare resource utilization and mortality through treatment benefits in the less severe hospitalized patients. Future combinations of treatments which impact different aspects of resource use may be optimal in reducing COVID-19-related pressure on healthcare system capacity. Policies that incentivize innovation and ensure equitable access to hospital care, testing, and treatment will be important to facilitate this.

\section{ACKNOWLEDGEMENTS}

Funding. No external funding or sponsorship was received for this study or publication of this article. The Rapid Service and Open Access Fees were funded by Genentech.
Authorship. All named authors meet the International Committee of Medical Journal Editors (ICMJE) criteria for authorship for this article, take responsibility for the integrity of the work as a whole, and have given their approval for this version to be published.

Disclosures. Daniel Sheinson, Caroline Solon, William Wong, and Anuj Shah are all salaried employees of Genentech; Mindy Cheng is a salaried employee of Roche Molecular Systems, Inc. David Elsea and Yang Meng received payment from Genentech for consulting fees. Daniel Sheinson, Caroline Solon, William Wong, and Mindy Cheng all hold Roche stock.

Compliance with Ethics Guidelines. The model described in this article leverages data from previously conducted studies and does not contain any studies with human participants or animals performed by any of the authors.

Data Availability. The datasets generated during and/or analyzed during the current study are available in the COVID Tracking Project repository, https://covidtracking.com/data/ api.

Open Access. This article is licensed under a Creative Commons Attribution-NonCommercial 4.0 International License, which permits any non-commercial use, sharing, adaptation, distribution and reproduction in any medium or format, as long as you give appropriate credit to the original author(s) and the source, provide a link to the Creative Commons licence, and indicate if changes were made. The images or other third party material in this article are included in the article's Creative Commons licence, unless indicated otherwise in a credit line to the material. If material is not included in the article's Creative Commons licence and your intended use is not permitted by statutory regulation or exceeds the permitted use, you will need to obtain permission directly from the copyright holder. To view a copy of this licence, visit http://creativecommons.org/licenses/bync/4.0/. 


\section{REFERENCES}

1. John Hopkins University \& Medicine. COVID-19 Dashboard. 2020. https://coronavirus.jhu.edu/map. html. Accessed 10 Aug 2020.

2. Wu Z, McGoogan JM. Characteristics of and important lessons from the coronavirus disease 2019 (COVID-19) outbreak in China: summary of a report of 72314 cases from the Chinese Center for Disease Control and Prevention. JAMA. 2020;323(13):1239-42.

3. Kim L, Garg S, O'Halloran A, et al. Risk factors for intensive care unit admission and in-hospital mortality among hospitalized adults identified through the U.S. Coronavirus Disease 2019 (COVID-19)-Associated Hospitalization Surveillance Network (COVID-NET). Clin Infect Dis. 2020;323(13): 1239-42.

4. Adelman D. Thousands of lives could be saved in the US during the COVID-19 pandemic if states exchanged ventilators. Health Aff (Millwood). 2020;39(7):1247-52.

5. Emanuel EJ, Persad G, Upshur R, et al. Fair allocation of scarce medical resources in the time of Covid-19. N Engl J Med. 2020;382(21):2049-55.

6. Moghadas SM, Shoukat A, Fitzpatrick MC, et al. Projecting hospital utilization during the COVID19 outbreaks in the United States. Proc Natl Acad Sci USA. 2020;117(16):9122-6.

7. Uppal A, Silvestri DM, Siegler M, et al. Critical care and emergency department response at the epicenter of the COVID-19 pandemic. Health Aff (Millwood). 2020;39(8):1443-9.

8. Argenziano MG, Bruce SL, Slater CL, et al. Characterization and clinical course of 1000 patients with coronavirus disease 2019 in New York: retrospective case series. Br Med J. 2020;369:m1996.

9. Cummings MJ, Baldwin MR, Abrams D, et al. Epidemiology, clinical course, and outcomes of critically ill adults with COVID-19 in New York City: a prospective cohort study. Lancet. 2020;395(10239): 1763-70.

10. Seneff MG, Zimmerman JE, Knaus WA, Wagner DP, Draper EA. Predicting the duration of mechanical ventilation. The importance of disease and patient characteristics. Chest. 1996;110(2):469-79.

11. Aiken LH, Clarke SP, Sloane DM, Sochalski J, Silber JH. Hospital nurse staffing and patient mortality, nurse burnout, and job dissatisfaction. JAMA. 2002;288(16):1987-93.
12. Coster $\mathrm{S}$. What is the impact of professional nursing on patients' outcomes globally? An overview of research evidence. Int J Nurs Stud. 2018;78:76-83.

13. Gupta S, Hayek SS, Wang W, et al. Factors associated with death in critically ill patients with coronavirus disease 2019 in the US. JAMA Intern Med. 2020;180(11):1436-46.

14. Lee A, Cheung YSL, Joynt GM, Leung CCH, Wong WT, Gomersall CD. Are high nurse workload/staffing ratios associated with decreased survival in critically ill patients? A cohort study. Ann Intensive Care. 2017;7(1):46.

15. Weissman GE, Crane-Droesch A, Chivers C, et al. Locally informed simulation to predict hospital capacity needs during the COVID-19 pandemic. Ann Intern Med. 2020;173(1):21-8.

16. National Institutes of Health. Accelerating COVID19 therapeutic innovations and vaccines (ACTIV). $2020 . \quad$ https://www.nih.gov/research-training/ medical-research-initiatives/activ. Accessed 9 Sep 2020 .

17. The COVID tracking project. Our data. 2020. https://covidtracking.com/data. Accessed 22 Aug 2020.

18. Mizumoto K, Kagaya K, Zarebski A, Chowell G. Estimating the asymptomatic proportion of coronavirus disease 2019 (COVID-19) cases on board the Diamond Princess cruise ship, Yokohama, Japan, 2020. Eurosurveillance. 2020;25(10):2000180.

19. World Health Organization. Report of the WHOChina Joint Mission on coronavirus disease 2019 (COVID-19). 2020. https://www.who.int/ publications/i/item/report-of-the-who-china-jointmission-on-coronavirus-disease-2019-(covid-19). Accessed 20 Apr 2020.

20. Liu J, West M. Combined parameter and state estimation in simulation-based filtering. In: Doucet A, de Freitas N, Gordon N, editors. Sequential Monte Carlo methods in practice. New York: Springer; 2001. p. 197-223.

21. R Core Team. R: a language and environment for statistical computing. 2019. https://www.R-project. org/.

22. Centers for Medicare \& Medicaid Services. CMSRuling 2020-1-R. 2020. https://www.cms.gov/files/ document/cms-2020-01-r.pdf. Accessed 10 Aug 2020.

23. Association for Molecular Pathology. SARS-CoV-2 molecular testing-summary of recent SARS-CoV-2 molecular testing survey. 2020. https://www.amp. 
org/AMP/assets/AMP_SARS-CoV-2_Survey_Report_ FINAL.pdf?pass=57. Accessed 14 Aug 2020.

24. Recovery Collaborative Group, Horby P, Lim WS, et al. Dexamethasone in hospitalized patients with Covid-19-preliminary report. N Engl J Med. 2020. https://doi.org/10.1056/NEJMoa2021436.

25. Beigel JH, Tomashek KM, Dodd LE. Remdesivir for the treatment of Covid-19-preliminary report. N Engl J Med. 2020;383(10):994.

26. Dyer O. Covid-19: remdesivir has little or no impact on survival, WHO trial shows. Br Med J. 2020;371: $\mathrm{m} 4057$.

27. WHO Solidarity trial consortium, Pan H, Peto R, et al. Repurposed antiviral drugs for COVID-19interim WHO SOLIDARITY trial results. medRxiv; 2020. p. 2020. https://doi.org/10.1101/2020.10.15. 20209817.

28. Healthcare Cost and Utilization Project. 2017 cost data for DRG 207 (Respiratory system diagnosis w ventilator support 96+ hours) and DRG 179 (Respiratory infections \& inflammations w/o cc/mcc). https://hcupnet.ahrq.gov/\#setup. Accessed 28 Aug 2020 .

29. Centers for Medicare \& Medicaid Services. Medicare Part D Drug Spending Dashboard \& Data. 2020. https://www.cms.gov/Research-Statistics-Data-andSystems/Statistics-Trends-and-Reports/ Information-on-Prescription-Drugs/MedicarePartD. Accessed 14 Sep 2020.

30. Gilead Sciences. Press release-an open letter from Daniel O-Day, Chairman \& CEO, Gilead Sciences. 2020. https://www.gilead.com/news-and-press/ press-room/press-releases/2020/6/an-open-letterfrom-daniel-oday-chairman-ceo-gilead-sciences. Accessed 29 Jun 2020.

31. National Institutes of Health. Coronavirus disease 2019 (COVID-19) treatment guidelines. 2020. https://www.covid19treatmentguidelines.nih.gov/. Accessed 21 Sept 2020.
32. American Clinical Laboratory Association. Press Release 07/14/2020_ACLA update on PCR testing capacity for Covid 19. 2020. https://www.acla.com/ acla-update-on-pcr-testing-capacity-for-covid-19/. Accessed 14 Sep 2020.

33. Milken Institute. COVID-19 treatment and vaccine tracker. 2020. https://covid-19tracker. milkeninstitute.org/. Accessed 28 Aug 2020.

34. Centers for Medicare \& Medicaid Services. FAQs about Families First Coronavirus Response Act and Coronavirus Aid, Relief, and Economic Security implementation Part 43. 2020. https://www.cms. gov/files/document/FFCRA-Part-43-FAQs.pdf. Accessed 26 Aug 2020.

35. Centers for Disease Control and Prevention. Health equity considerations and racial and ethnic minority groups. 2020. https://www.cdc.gov/ coronavirus/2019-ncov/community/health-equity/ race-ethnicity.html. Accessed 28 Aug 2020.

36. Krouse HJ. COVID-19 and the widening gap in health inequity. Otolaryngol Head Neck Surg. 2020;163(1):65-6.

37. Shadmi E, Chen Y, Dourado I, et al. Health equity and COVID-19: global perspectives. Int J Equity Health. 2020;19(1):104.

38. Hudman J, McDermott D, Kurani N, Cox C. Costsharing waivers and premium relief by private plans in response to COVID-19. 2020. https://www. healthsystemtracker.org/brief/cost-sharing-waiversand-premium-relief-by-private-plans-in-responseto-covid-19/. Accessed 28 Aug 2020.

39. Giammaria D, Pajewski A. Can early treatment of patients with risk factors contribute to managing the COVID-19 pandemic? J Glob Health. 2020;10(1):010377.

40. Sun Q, Qiu H, Huang M, Yang Y. Lower mortality of COVID-19 by early recognition and intervention: experience from Jiangsu Province. Ann Intensive Care. 2020;10(1):33. 